\title{
Analytical properties of type 2 degenerate poly-Bernoulli polynomials associated with their applications
}

\author{
Waseem A. Khan ${ }^{1 *} \mathbb{D}$, Ghulam Muhiuddin², Abdulghani Muhyi ${ }^{3}$ and Deena Al-Kadi ${ }^{4}$
}

\author{
"Correspondence: \\ wkhan1@pmu.edu.sa \\ 'Department of Mathematics and \\ Natural Sciences, Prince \\ Mohammad Bin Fahd University, P.O \\ Box 1664, Al Khobar 31952, Saudi \\ Arabia \\ Full list of author information is \\ available at the end of the article
}

\begin{abstract}
Recently, Kim et al. (Adv. Differ. Equ. 2020:168, 2020) considered the poly-Bernoulli numbers and polynomials resulting from the moderated version of degenerate polyexponential functions. In this paper, we investigate the degenerate type 2 poly-Bernoulli numbers and polynomials which are derived from the moderated version of degenerate polyexponential functions. Our degenerate type 2 degenerate poly-Bernoulli numbers and polynomials are different from those of Kim et al. (Adv. Differ. Equ. 2020:168, 2020) and Kim and Kim (Russ. J. Math. Phys. 26(1):40-49, 2019). Utilizing the properties of moderated degenerate poly-exponential function, we explore some properties of our type 2 degenerate poly-Bernoulli numbers and polynomials. From our investigation, we derive some explicit expressions for type 2 degenerate poly-Bernoulli numbers and polynomials. In addition, we also scrutinize type 2 degenerate unipoly-Bernoulli polynomials related to an arithmetic function and investigate some identities for those polynomials. In particular, we consider certain new explicit expressions and relations of type 2 degenerate unipoly-Bernoulli polynomials and numbers related to special numbers and polynomials. Further, some related beautiful zeros and graphical representations are displayed with the help of Mathematica.
\end{abstract}

MSC: 11B73; 11B83; 05A19

Keywords: Modified degenerate polyexponential functions; Type 2 degenerate Bernoulli polynomials; Type 2 degenerate central Bell polynomials; Unipoly functions

\section{黛 Springer}

\section{Introduction}

Carlitz [1, 2], Kim and Kim [14, 19, 21-23], Kim et al. [26, 28, 29, 31], Jang et al. [7, 8], Muhiuddin et al. [37-39], Khan et al. [10-13], Sharma et al. [41-43] introduced and studied various degenerate versions of special polynomials and numbers like degenerate Bernoulli polynomials, degenerate Euler polynomials, degenerate Daehee polynomials, degenerate Fubini polynomials, and degenerate Stirling numbers of the first and second kinds.

(c) The Author(s) 2021. This article is licensed under a Creative Commons Attribution 4.0 International License, which permits use, sharing, adaptation, distribution and reproduction in any medium or format, as long as you give appropriate credit to the original author(s) and the source, provide a link to the Creative Commons licence, and indicate if changes were made. The images or other third party material in this article are included in the article's Creative Commons licence, unless indicated otherwise in a credit line to the material. If material is not included in the article's Creative Commons licence and your intended use is not permitted by statutory regulation or exceeds the permitted use, you will need to obtain permission directly from the copyright holder. To view a copy of this licence, visit http://creativecommons.org/licenses/by/4.0/. 
For $\kappa \in \mathbb{Z}$, the modified degenerate polyexponential function [30] is specified by Kim and Kim to be

$$
\mathrm{Ei}_{\kappa, \lambda}(\omega)=\sum_{v=1}^{\infty} \frac{(1)_{v, \lambda} \omega^{v}}{(v-1) ! v^{\kappa}}, \quad(|\omega|<1)
$$

Note that

$$
\operatorname{Ei}_{1, \lambda}(\omega)=\sum_{v=1}^{\infty} \frac{(1)_{v, \lambda} \omega^{v}}{v !}=e_{\lambda}(\omega)-1
$$

The degenerate polyexponential functions were introduced by Kim and Kim as

$$
e_{\lambda}(\omega, \delta \mid \kappa)=\sum_{v=0}^{\infty} \frac{(1)_{v, \lambda} \omega^{v}}{v !(v+\delta)^{\kappa}} \quad(\text { see }[21])
$$

where $\delta \in \mathbb{C}$ and $\kappa \in \mathbb{N} \cup\{0\}$ with $\mathfrak{R}(\delta)>0$.

From (1.3), we readily get

$$
e_{\lambda}(\omega, \delta \mid 0)=e_{\lambda}(\omega), \quad e_{\xi}(\omega, 1 \mid 1)=\frac{1}{\omega} \frac{1}{1+\xi}\left(e_{\lambda}(\omega)-1\right)+\frac{\lambda}{1+\lambda} e_{\lambda}(\omega)
$$

We note here that

$$
\lim _{\lambda \rightarrow 0} e_{\lambda}(\omega, 1 \mid 1)=\frac{1}{\omega}\left(e^{\omega}-1\right)=e(\omega, 1 \mid 1)
$$

which was defined by Hardy (see $[5,6])$.

The degenerate poly-Genocchi polynomials [30] were considered by Kim and Kim and given as

$$
\frac{2 \mathrm{Ei}_{\kappa, \lambda}\left(\log _{\lambda}(1+z)\right)}{e_{\lambda}(z)+1} e_{\lambda}^{\omega}(z)=\sum_{v=0}^{\infty} G_{v, \lambda}^{(\kappa)}(\omega) \frac{z^{v}}{v !} \quad(\kappa \in \mathbb{Z}) .
$$

When $\omega=0, G_{v, \lambda}^{(\kappa)}=G_{v, \lambda}^{(\kappa)}(0)$ are called degenerate poly-Genocchi numbers.

From (1.1) and (1.5), we see that $G_{v, \lambda}^{(1)}(\omega)=G_{v, \lambda}(\omega),(v \geq 0)$ which are called degenerate Genocchi polynomials.

For $\lambda \in \mathbb{R}$, the degenerate exponential function is defined as follows (see [14-16, 1834]):

$$
e_{\lambda}^{\omega}(z)=(1+\lambda z)^{\frac{\omega}{\lambda}}, \quad e_{\lambda}(z):=e_{\lambda}^{1}(z)=(1+\lambda z)^{\frac{1}{\lambda}}
$$

and

$$
e_{\lambda}^{\omega}(z)=\sum_{v=0}^{\infty}(\omega)_{v, \lambda} \frac{z^{v}}{v !}
$$

where $(\omega)_{0, \lambda}=1,(\omega)_{v, \lambda}=\omega(\omega-\lambda)(\omega-2 \lambda) \cdots(\omega-(v-1) \lambda),(v \geq 1)$. 
The degenerate Bernoulli polynomials considered by Carlitz $[1,2]$ are given by

$$
\frac{z}{e_{\lambda}(z)-1} e_{\lambda}^{\omega}(z)=\sum_{v=0}^{\infty} \beta_{v, \lambda}(\omega) \frac{z^{v}}{v !}
$$

When $\omega=0, \beta_{v, \lambda}=\beta_{v, \lambda}(0)$ denotes degenerate Bernoulli numbers.

In 2019, Jang and Kim [7] introduced type 2 degenerate Bernoulli polynomials as follows:

$$
\frac{z}{e_{\lambda}^{\frac{1}{2}}(z)-e_{\lambda}^{-\frac{1}{2}}(z)} e_{\lambda}^{\omega}(z)=\sum_{v=0}^{\infty} B_{v, \lambda}(\omega) \frac{z^{v}}{v !}
$$

When $\omega=0, B_{v, \lambda}=B_{v, \lambda}(0)$ are type 2 degenerate Bernoulli numbers.

We note that

$$
\begin{aligned}
\lim _{\lambda \rightarrow 0} \frac{z}{e_{\lambda}^{\frac{1}{2}}(z)-e_{\lambda}^{-\frac{1}{2}}(z)} e_{\lambda}^{\omega}(z) & =\sum_{v=0}^{\infty} \lim _{\lambda \rightarrow 0} B_{v, \lambda}(\omega) \frac{z^{v}}{v !} \\
& =\frac{z}{e^{\frac{z}{2}}-e^{-\frac{z}{2}}} e^{\omega z}=\sum_{v=0}^{\infty} B_{v}(\omega) \frac{z^{v}}{v !}
\end{aligned}
$$

are called type 2 Bernoulli polynomials $B_{v}(\omega),(v \geq 0)$.

The degenerate form of central Bell polynomials is given as (see [20])

$$
e^{\omega\left(e_{\lambda}^{\frac{1}{2}}(z)-e_{\lambda}^{-\frac{1}{2}}(z)\right)}=\sum_{v=0}^{\infty} B e l_{v, \lambda}^{(c)}(\omega) \frac{z^{v}}{v !}
$$

For $\omega=1, B e l_{v, \lambda}^{(c)}=B e l_{v, \lambda}^{(c)}(1)$ denotes degenerate central Bell numbers.

For $\lambda \in \mathbb{R}, \mathrm{Kim}$ and Kim [15] defined the degenerate version of the logarithm function denoted by $\log _{\lambda}(1+t)$ as follows:

$$
\log _{\lambda}(1+z)=\sum_{v=1}^{\infty} \lambda^{v-1}(1)_{v, 1 / \lambda} \frac{z^{v}}{v !}
$$

being the inverse of the degenerate version of the exponential function $e_{\lambda}(z)$ as has been shown below:

$$
e_{\lambda}\left(\log _{\lambda}(z)\right)=\log _{\lambda}\left(e_{\lambda}(z)\right)=z
$$

It is noteworthy to mention that

$$
\lim _{\lambda \rightarrow 0} \log _{\lambda}(1+z)=\sum_{v=1}^{\infty}(-1)^{v-1} \frac{z^{v}}{v !}=\log (1+z) .
$$

The degenerate Daehee polynomials of order $r$ are defined by (see $[28,41])$

$$
\left(\frac{\log _{\lambda}(1+z)}{z}\right)^{r}(1+z)^{\omega}=\sum_{v=0}^{\infty} D_{v, \lambda}^{(r)}(\omega) \frac{z^{v}}{v !} .
$$


If $\omega=0, D_{v, \lambda}^{(r)}=D_{v, \lambda}^{(r)}(0),(v \geq 0)$ denotes degenerate Daehee numbers of order $r$. For $r=1,(1.13)$ reduces to

$$
\frac{\log _{\lambda}(1+z)}{z}(1+z)^{\omega}=\sum_{v=0}^{\infty} D_{v, \lambda}(\omega) \frac{z^{v}}{v !}
$$

If $\omega=0, D_{v, \lambda}=D_{v, \lambda}(0),(v \geq 0)$ denotes degenerate Daehee numbers.

The degenerate Bernoulli polynomials of the second kind are specified by

$$
\frac{z}{\log _{\lambda}(1+z)}(1+z)^{\omega}=\sum_{v=0}^{\infty} b_{v, \lambda}(\omega) \frac{z^{v}}{v !} \quad(\text { see }[18])
$$

When $\omega=0, b_{v, \lambda}=b_{v, \lambda}(0)(v \geq 0)$ denotes degenerate Bernoulli numbers of the second kind.

The degenerate form of first kind Stirling numbers is defined by

$$
\frac{1}{\kappa !}\left(\log _{\lambda}(1+z)\right)^{\kappa}=\sum_{v=\kappa}^{\infty} S_{1, \lambda}(v, \kappa) \frac{z^{v}}{v !} \quad(\kappa \geq 0),(\text { see }[21,30]) .
$$

Note here that $\lim _{\lambda \rightarrow 0} S_{1, \lambda}(v, \kappa)=S_{1}(v, \kappa)$, where $S_{1}(v, \kappa)$ are first kind Stirling numbers given by

$$
\frac{1}{\kappa !}(\log (1+z))^{\kappa}=\sum_{v=\kappa}^{\infty} S_{1}(v, \kappa) \frac{z^{v}}{v !} \quad(\kappa \geq 0),(\text { see }[1-14,17,21,26,28]) .
$$

The degenerate form of second kind Stirling numbers is defined by

$$
\frac{1}{\kappa !}\left(e_{\lambda}(z)-1\right)^{\kappa}=\sum_{v=\kappa}^{\infty} S_{2, \lambda}(v, \kappa) \frac{z^{v}}{v !} \quad(\text { see }[17])
$$

Here, $\lim _{\lambda \rightarrow 0} S_{2, \lambda}(v, \kappa)=S_{2}(v, \kappa)$, where $S_{2}(v, \kappa)$ are second kind Stirling numbers given by

$$
\frac{1}{\kappa !}\left(e^{z}-1\right)^{\kappa}=\sum_{v=\kappa}^{\infty} S_{2}(v, \kappa) \frac{z^{v}}{v !} \quad(\text { see }[1-43]) .
$$

The degenerate form of second kind central factorial polynomials [3] is given as

$$
\frac{1}{\kappa !}\left(e_{\lambda}^{\frac{1}{2}}(z)-e_{\lambda}^{-\frac{1}{2}}(z)\right)^{\kappa} e_{\lambda}^{\omega}(z)=\sum_{j=\kappa}^{\infty} T_{2, \lambda}(j, \kappa \mid \omega) \frac{z^{j}}{j !} \quad(\kappa \geq 0)
$$

If $\omega=0, T_{2, \lambda}(j, \kappa)=T_{2, \lambda}(j, \kappa \mid 0)$ denotes second kind degenerate central factorial numbers.

This article aims to present type 2 degenerate poly-Bernoulli numbers and polynomials arising from moderated degenerate polyexponential functions. Certain explicit expressions for these numbers and polynomials are derived. Also, we introduce type 2 degenerate unipoly-Bernoulli numbers and polynomials by utilizing unipoly functions and show some basic properties of them. 


\section{Type $\mathbf{2}$ degenerate poly-Bernoulli polynomials and numbers}

For $\kappa \in \mathbb{Z}$, and utilizing the modified degenerate polyexponential functions, we consider type 2 degenerate poly-Bernoulli polynomials as

$$
\frac{\mathrm{Ei}_{\kappa, \lambda}\left(\log _{\lambda}(1+z)\right)}{e_{\lambda}^{\frac{1}{2}}(z)-e_{\lambda}^{-\frac{1}{2}}(z)} e_{\lambda}^{\omega}(z)=\sum_{v=0}^{\infty} \beta_{v, \lambda}^{(\kappa)}(\omega) \frac{z^{v}}{v !} .
$$

If $\omega=0, \beta_{v, \lambda}^{(\kappa)}=\beta_{v, \lambda}^{(\kappa)}(0)$ denotes type 2 degenerate poly-Bernoulli numbers.

For $\kappa=1$, by using (2.1) and (1.2), we see that

$$
\frac{\mathrm{Ei}_{1, \lambda}\left(\log _{\lambda}(1+z)\right)}{e_{\lambda}^{\frac{1}{2}}(z)-e_{\lambda}^{-\frac{1}{2}}(z)} e_{\lambda}^{\omega}(z)=\frac{z}{e_{\lambda}^{\frac{1}{2}}(z)-e_{\lambda}^{-\frac{1}{2}}(z)} e_{\lambda}^{\omega}(z)=\sum_{v=0}^{\infty} B_{v, \lambda}(\omega) \frac{z^{v}}{v !} \quad(v \geq 0),
$$

where $B_{v, \lambda}(\omega)$ are called type 2 degenerate Bernoulli polynomials (see [3]).

First, we can write that

$$
\begin{aligned}
\operatorname{Ei}_{\kappa, \lambda}\left(\log _{\lambda}(1+z)\right) & =\sum_{i=1}^{\infty} \frac{(1)_{i, \lambda}\left(\log _{\lambda}(1+z)\right)^{i}}{(i-1) ! i^{\kappa}} \\
& =\sum_{i=1}^{\infty} \frac{(1)_{i, \lambda}}{i^{\kappa-1}} \frac{\left(\log _{\lambda}(1+z)\right)^{i}}{i !} \\
& =\sum_{i=1}^{\infty} \frac{(1)_{i, \lambda}}{i^{\kappa-1}} \sum_{v=i}^{\infty} S_{1, \lambda}(v, i) \frac{z^{v}}{v !} \\
& =\sum_{v=1}^{\infty}\left(\sum_{i=1}^{v} \frac{(1)_{i, \lambda}}{i^{\kappa-1}} S_{1, \lambda}(v, i)\right) \frac{z^{v}}{v !} .
\end{aligned}
$$

By (2.3), we see that (2.1) is equal to

$$
\begin{aligned}
\frac{\mathrm{Ei}_{\kappa, \lambda}\left(\log _{\lambda}(1+z)\right)}{e_{\lambda}^{\frac{1}{2}}(z)-e_{\lambda}^{-\frac{1}{2}}(z)} & =\frac{1}{e_{\lambda}^{\frac{1}{2}}(z)-e_{\lambda}^{-\frac{1}{2}}(z)} \sum_{r=1}^{\infty}\left(\sum_{i=1}^{r} \frac{(1)_{i, \lambda}}{i^{\kappa-1}} S_{1, \lambda}(r, i)\right) \frac{z^{r}}{r !} \\
& =\frac{z}{e_{\lambda}^{\frac{1}{2}}(z)-e_{\lambda}^{-\frac{1}{2}}(z)} \sum_{r=0}^{\infty}\left(\sum_{i=1}^{r+1} \frac{(1)_{i, \lambda}}{i^{\kappa-1}} \frac{S_{1, \lambda}(r+1, i)}{r+1}\right) \frac{z^{r}}{r !} \\
& =\sum_{v=0}^{\infty} B_{v, \lambda} \frac{z^{v}}{v !} \sum_{r=0}^{\infty}\left(\sum_{i=1}^{r+1} \frac{(1)_{i, \lambda}}{i^{\kappa-1}} \frac{S_{1, \lambda}(r+1, i)}{l+1}\right) \frac{z^{r}}{r !} \\
& =\sum_{v=0}^{\infty}\left(\sum_{r=0}^{v}\left(\begin{array}{l}
v \\
r
\end{array}\right) \sum_{i=1}^{r+1} \frac{\left.(1)_{i, \lambda} \frac{S_{1, \lambda}(r+1, i)}{i^{\kappa-1}} B_{v-l, \lambda}\right) \frac{z^{v}}{v !} .}{}\right.
\end{aligned}
$$

Therefore, by (2.1) and (2.4), we arrive at the following theorem.

Theorem 2.1 For $\kappa \in \mathbb{Z}$ and $v \geq 0$, we have

$$
\beta_{v, \lambda}^{(\kappa)}=\sum_{r=0}^{v}\left(\begin{array}{l}
v \\
r
\end{array}\right) \sum_{i=1}^{r+1} \frac{(1)_{i, \lambda}}{i^{\kappa-1}} \frac{S_{1, \lambda}(r+1, i)}{l+1} B_{v-r, \lambda}
$$


Corollary 2.1 Putting $k=1$ in Theorem 2.1 yields

$$
\beta_{v, \lambda}=\sum_{r=0}^{v}\left(\begin{array}{l}
v \\
r
\end{array}\right) \sum_{i=1}^{r+1} \frac{S_{1, \lambda}(r+1, i)}{r+1}(1)_{i, \lambda} B_{v-r, \lambda}
$$

Remark 2.1 Letting $\lambda$ to 0 in Theorem 2.1 leads to

$$
\beta_{v}^{(\kappa)}=\sum_{r=0}^{v}\left(\begin{array}{l}
v \\
r
\end{array}\right) \sum_{i=1}^{r+1} \frac{S_{1}(r+1, i)}{i^{\kappa-1} r+1} B_{v-r} \quad(v \geq 0)
$$

Using (1.1), we have

$$
\begin{aligned}
\frac{\mathrm{d}}{\mathrm{dz}} \mathrm{Ei}_{\kappa, \lambda}\left(\log _{\lambda}(1+z)\right) & =\frac{\mathrm{d}}{\mathrm{dz}} \sum_{v=1}^{\infty} \frac{(1)_{v, \lambda}\left(\log _{\lambda}(1+z)\right)^{v}}{(v-1) ! v^{\kappa}} \\
& =\frac{(1+z)^{\lambda-1}}{\log _{\lambda}(1+z)} \sum_{v=1}^{\infty} \frac{(1)_{v, \lambda}\left(\log _{\lambda}(1+z)\right)^{v}}{(v-1) ! v^{\kappa-1}} \\
& =\frac{(1+z)^{\lambda-1}}{\log _{\lambda}(1+z)} \mathrm{Ei}_{\kappa-1, \lambda}\left(\log _{\lambda}(1+z)\right) .
\end{aligned}
$$

By (2.5), for $\kappa \geq 2$, we have

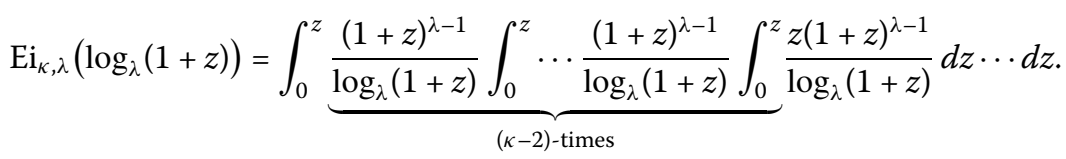

Then, from (2.1) and (2.6), we have

$$
\begin{aligned}
\sum_{v=0}^{\infty} \beta_{v, \lambda}^{(\kappa)} \frac{z^{v}}{v !}= & \frac{1}{e_{\lambda}^{\frac{1}{2}}(z)-e_{\lambda}^{-\frac{1}{2}}(z)} \operatorname{Ei}_{\kappa, \lambda}\left(\log _{\lambda}(1+z)\right) \\
= & \frac{1}{e_{\lambda}^{\frac{1}{2}}(z)-e_{\lambda}^{-\frac{1}{2}}(z)} \int_{0}^{z} \underbrace{\frac{(1+z)^{\lambda-1}}{\log _{\lambda}(1+z)} \int_{0}^{z} \cdots \frac{(1+z)^{\lambda-1}}{\log _{\lambda}(1+z)} \int_{0}^{z} \frac{z(1+z)^{\lambda-1}}{\log _{\lambda}(1+z)} d z \cdots d z}_{(\kappa-2) \text {-times }} \\
= & \frac{z}{e_{\lambda}^{\frac{1}{2}}(z)-e_{\lambda}^{-\frac{1}{2}}(z)} \sum_{i=0}^{\infty} \sum_{i_{1}+\cdots+i_{\kappa-1}=i}\left(\begin{array}{c}
i \\
i_{1}+\cdots+i_{\kappa-1}
\end{array}\right) \\
& \times \frac{b_{i_{1}, \lambda}(\lambda-1)}{i_{1}+1} \frac{b_{i_{2}, \lambda}(\lambda-1)}{i_{1}+i_{2}+1} \cdots \frac{b_{i_{\kappa-1}, \lambda}(\lambda-1)}{i_{1}+\cdots+i_{\kappa-1}+1} \frac{z^{i}}{i !} \\
= & \sum_{v=0}^{\infty} \sum_{i=0}^{v}\left(\begin{array}{l}
v \\
i
\end{array}\right) \\
& \times \frac{b_{i_{1}, \lambda}(\lambda-1)}{i_{1}+1} \frac{\sum_{i_{1}+\cdots+i_{\kappa-1}=i}(\lambda-1)}{i_{1}+i_{2}+1} \cdots \frac{b_{i_{\kappa-1}, \lambda}(\lambda-1)}{i_{1}+\cdots+i_{\kappa-1}+1} B_{v-i, \lambda} \frac{z^{v}}{v !}
\end{aligned}
$$

Therefore, by (2.7), we arrive at the following theorem. 
Theorem 2.2 For $v \geq 0$, then

$$
\begin{aligned}
\beta_{v, \lambda}^{(\kappa)}= & \sum_{i=0}^{v}\left(\begin{array}{c}
v \\
i
\end{array}\right) \sum_{i_{1}+\cdots+i_{\kappa-1}=i}\left(\begin{array}{c}
i \\
i_{1}+\cdots+i_{\kappa-1}
\end{array}\right) \\
& \times \frac{b_{i_{1}, \lambda}(\lambda-1)}{i_{1}+1} \frac{b_{i_{2}, \lambda}(\lambda-1)}{i_{1}+i_{2}+1} \cdots \frac{b_{i_{\kappa-1}, \lambda}(\lambda-1)}{i_{1}+\cdots+i_{\kappa-1}+1} B_{v-i, \lambda} .
\end{aligned}
$$

By making use of (1.7) and (2.1), we note that

$$
\begin{aligned}
\sum_{v=0}^{\infty} \beta_{v, \lambda}^{(\kappa)}(\omega) \frac{z^{v}}{v !} & =\frac{\operatorname{Ei}_{\kappa, \lambda}\left(\log _{\lambda}(1+z)\right)}{e_{\lambda}^{\frac{1}{2}}(z)-e_{\lambda}^{-\frac{1}{2}}(z)} e_{\lambda}^{\omega}(z) \\
& =\sum_{r=0}^{\infty} \beta_{r, \lambda}^{(\kappa)} \frac{z^{r}}{r !} \sum_{v=0}^{\infty}(\omega)_{v, \lambda} \frac{z^{v}}{v !} \\
& =\sum_{v=0}^{\infty}\left(\sum_{r=0}^{v}\left(\begin{array}{l}
v \\
r
\end{array}\right) \beta_{r, \lambda}^{(\kappa)}(\omega)_{v-r, \lambda}\right) \frac{z^{v}}{v !},
\end{aligned}
$$

which on comparing the coefficients on both sides of the above equation yields the following theorem.

Theorem 2.3 For $v \geq 0$, we have

$$
\beta_{v, \lambda}^{(\kappa)}(\omega)=\sum_{r=0}^{v}\left(\begin{array}{l}
v \\
r
\end{array}\right) \beta_{r, \lambda}^{(\kappa)}(\omega)_{v-r, \lambda}
$$

Let $\kappa \geq 1$ be an integer. For $s \in \mathbb{C}$, the function $\eta_{\kappa, \lambda}(s)$ can be defined as

$$
\begin{aligned}
\eta_{\kappa, \lambda}(s)= & \frac{1}{\Gamma(s)} \int_{0}^{\infty} \frac{z^{s-1}}{e_{\lambda}^{\frac{1}{2}}(z)-e_{\lambda}^{-\frac{1}{2}}(z)} \operatorname{Ei}_{\kappa, \lambda}\left(\log _{\lambda}(1+z)\right) d z \\
= & \frac{1}{\Gamma(s)} \int_{0}^{1} \frac{z^{s-1}}{e_{\lambda}^{\frac{1}{2}}(z)-e_{\lambda}^{-\frac{1}{2}}(z)} \operatorname{Ei}_{\kappa, \lambda}\left(\log _{\lambda}(1+z)\right) d z \\
& +\frac{1}{\Gamma(s)} \int_{1}^{\infty} \frac{z^{s-1}}{e_{\lambda}^{\frac{1}{2}}(z)-e_{\lambda}^{-\frac{1}{2}}(z)} \operatorname{Ei}_{\kappa, \lambda}\left(\log _{\lambda}(1+z)\right) d z
\end{aligned}
$$

Here, for any $s \in \mathbb{C}$, the second integral converges absolutely; hence, the second term on the r.h.s. vanishes at nonpositive integers, i.e.,

$$
\lim _{s \rightarrow-n}\left|\frac{1}{\Gamma(s)} \int_{1}^{\infty} \frac{z^{s-1}}{e_{\lambda}^{\frac{1}{2}}(z)-e_{\lambda}^{-\frac{1}{2}}(z)} \operatorname{Ei}_{\kappa, \lambda}\left(\log _{\lambda}(1+z)\right) d z\right| \leq \frac{1}{\Gamma(-n)} N=0 .
$$

On the other hand, for $\Re(s)>0$, we can write the first integral in (2.10) as

$$
\frac{1}{\Gamma(s)} \sum_{r=0}^{\infty} \frac{\beta_{r, \lambda}^{(\kappa)}}{r !} \frac{1}{s+r}
$$

which defines an entire function of $s$. Therefore, we may say that $\eta_{\kappa, \lambda}(s)$ can be continued to an entire function of $s$. 
Further, from (2.10) and (2.11), we get

$$
\begin{aligned}
\eta_{\kappa, \lambda}(-n) & =\lim _{s \rightarrow-n} \frac{1}{\Gamma(s)} \int_{0}^{1} \frac{z^{s-1}}{e_{\lambda}^{\frac{1}{2}}(z)-e_{\lambda}^{-\frac{1}{2}}(z)} \operatorname{Ei}_{\kappa, \lambda}\left(\log _{\lambda}(1+z)\right) d z \\
& =\lim _{s \rightarrow-n} \frac{1}{\Gamma(s)} \int_{0}^{1} z^{s-1} \sum_{r=0}^{\infty} \frac{B_{r, \lambda}^{(\kappa)} z^{r}}{r !} d z=\lim _{s \rightarrow-n} \frac{1}{\Gamma(s)} \sum_{r=0}^{\infty} \frac{\beta_{r, \lambda}^{(\kappa)}}{s+r} \frac{1}{r !} \\
& =\cdots+0+\cdots+0+\lim _{s \rightarrow-n} \frac{1}{\Gamma(s)} \frac{1}{s+n} \frac{\beta_{n, \lambda}^{(\kappa)}}{n !}+0+0+\cdots \\
& =\lim _{s \rightarrow-n} \frac{\left(\frac{\Gamma(1-s) \sin \pi s}{\pi}\right)}{s+n} \frac{\beta_{n, \lambda}^{(\kappa)}}{n !}=\Gamma(1+n) \cos (\pi n) \frac{\beta_{n, \lambda}^{(\kappa)}}{n !} \\
& =(-1)^{n} \beta_{n, \lambda}^{(\kappa)} .
\end{aligned}
$$

Therefore, by (2.12), we arrive at the following theorem.

Theorem 2.4 Let $\kappa \geq 1$ and $n \in \mathbb{N} \cup\{0\}, s \in \mathbb{C}$. Then

$$
\eta_{\kappa, \lambda}(-n)=(-1)^{n} \beta_{n, \lambda}^{(\kappa)}
$$

By making use of (1.2), we note that

$$
\begin{aligned}
\operatorname{Ei}_{1, \lambda}\left(\log _{\lambda}(1+z)\right) & =\sum_{i=1}^{\infty} \frac{(1)_{i, \lambda}\left(\log _{\lambda}(1+z)\right)^{i}}{i !} \\
& =\sum_{i=1}^{\infty} \frac{(1)_{i, \lambda}\left(\log _{\lambda}(1+z)\right)^{i}}{i !} \\
& =\sum_{i=1}^{\infty} \sum_{v=i}^{\infty}(1)_{i, \lambda} S_{1, \lambda}(v, i) \frac{z^{v}}{v !} \\
& =\sum_{v=1}^{\infty}\left(\sum_{i=1}^{v}(1)_{i, \lambda} S_{1, \lambda}(v, i)\right) \frac{z^{v}}{v !}
\end{aligned}
$$

On the other hand,

$$
\operatorname{Ei}_{1, \lambda}\left(\log _{\lambda}(1+z)\right)=z
$$

Therefore, by (2.13) and (2.14), we arrive at the following theorem.

Theorem 2.5 For $v \in \mathbb{N}$, then

$$
\sum_{i=1}^{v}(1)_{i, \lambda} S_{1, \lambda}(v, i)=\delta_{v, 1} \text {, }
$$

where $\delta_{v, \kappa}$ is the Kronecker delta. 
From (2.1), we note that

$$
\frac{\operatorname{Ei}_{\kappa, \lambda}\left(\log _{\lambda}(1+z)\right)}{e_{\lambda}^{\frac{1}{2}}(z)-e_{\lambda}^{-\frac{1}{2}}(z)}=\sum_{v=0}^{\infty} \beta_{v, \lambda}^{(\kappa)} \frac{z^{v}}{v !}
$$

On replacing $z$ with $e_{\lambda}(z)-1$ in (2.15), we get

$$
\begin{aligned}
\sum_{m=0}^{\infty} & \beta_{m, \lambda}^{(\kappa)} \frac{\left(e_{\lambda}(z)-1\right)^{m}}{m !} \\
= & \frac{\operatorname{Ei}_{\kappa, \lambda}(z)}{e_{\lambda}^{\frac{1}{2}}\left(e_{\lambda}(z)-1\right)-e_{\lambda}^{-\frac{1}{2}}\left(e_{\lambda}(z)-1\right)} \\
= & \frac{e_{\lambda}(z)-1}{e_{\lambda}^{\frac{1}{2}}\left(e_{\lambda}(z)-1\right)-e_{\lambda}^{-\frac{1}{2}}\left(e_{\lambda}(z)-1\right)} \frac{z}{e_{\lambda}(z)-1} \frac{\mathrm{Ei}_{\kappa, \lambda}(z)}{z} \\
= & \sum_{i=0}^{\infty} B_{i, \lambda} \frac{1}{i !}\left(e_{\lambda}(z)-1\right)^{i} \sum_{j=0}^{\infty} B_{j, \lambda} \frac{z^{j}}{j !} \sum_{v=0}^{\infty} \frac{(1)_{v+1, \lambda} z^{v}}{v !(v+1)^{\kappa}} \\
= & \sum_{i=0}^{\infty} B_{i, \lambda} \sum_{l=i}^{\infty} S_{2, \lambda}(l, i) \frac{z^{l}}{l !} \sum_{j=0}^{\infty} B_{j, \lambda} \frac{z^{j}}{j !} \sum_{v=0}^{\infty} \frac{(1)_{v+1, \lambda} z^{v}}{v !(v+1)^{\kappa}} \\
= & \sum_{l=0}^{\infty} \sum_{i=0}^{l} B_{i, \lambda} S_{2, \lambda}(l, i) \frac{z^{l}}{l !} \sum_{j=0}^{\infty} B_{j, \lambda} \frac{z^{j}}{j !} \sum_{v=0}^{\infty} \frac{(1)_{v+1, \lambda} z^{v}}{v !(v+1)^{\kappa}} \\
= & \sum_{j=0}^{\infty}\left(\sum _ { l = 0 } ^ { j } \sum _ { i = 0 } ^ { l } \left(\sum_{l=0}^{\infty}\left(\sum_{j=0}^{v} \sum_{l, \lambda}^{j} S_{l=0}^{j} \sum_{i=0}^{l}(l, i) B_{j-l, \lambda}\right) \frac{z^{j}}{j !} \times \sum_{v=0}^{\infty} \frac{(1)_{v+1, \lambda} z^{v}}{v !(v+1)^{\kappa}}\right.\right. \\
j & \left.B_{i, \lambda} S_{2, \lambda}(l, i) B_{j-l, \lambda} \frac{(1)_{v-j+1, \lambda}}{(v-j+1)^{\kappa}}\right) \frac{z^{v}}{v !} .
\end{aligned}
$$

On the other hand,

$$
\begin{aligned}
\sum_{m=0}^{\infty} \beta_{m, \lambda}^{(\kappa)} \frac{\left(e_{\lambda}(z)-1\right)^{m}}{m !} & =\sum_{m=0}^{\infty} \beta_{m, \lambda}^{(\kappa)} \sum_{v=m}^{\infty} S_{2, \lambda}(v, m) \frac{z^{v}}{v !} \\
& =\sum_{v=0}^{\infty}\left(\sum_{m=0}^{v} \beta_{m, \lambda}^{(\kappa)} S_{2, \lambda}(v, m)\right) \frac{z^{v}}{v !}
\end{aligned}
$$

Therefore, by (2.16) and (2.17), we arrive at the following theorem.

Theorem 2.6 For $v \in \mathbb{N}$, we have

$$
\sum_{m=0}^{v} \beta_{m, \lambda}^{(\kappa)} S_{2, \lambda}(v, m)=\sum_{j=0}^{v} \sum_{l=0}^{j} \sum_{i=0}^{l}\left(\begin{array}{l}
j \\
l
\end{array}\right)\left(\begin{array}{l}
v \\
j
\end{array}\right) B_{i, \lambda} S_{2, \lambda}(l, i) B_{j-l, \lambda} \frac{(1)_{v-j+1, \lambda}}{(v-j+1)^{\kappa}} .
$$


The higher-order type 2 degenerate Bernoulli polynomials of order $r \in \mathbb{N}$ are defined by (see [7])

$$
\left(\frac{z}{e_{\lambda}^{\frac{1}{2}}(z)-e_{\lambda}^{-\frac{1}{2}}(z)}\right)^{r} e_{\lambda}^{\omega}(z)=\sum_{v=0}^{\infty} B_{v, \lambda}^{(r)}(\omega) \frac{z^{v}}{v !} .
$$

For $\omega=0, B_{v, \lambda}^{(r)}(0)=B_{v, \lambda}^{(r)}$ denotes higher-order type 2 degenerate Bernoulli numbers of order $r \in \mathbb{N}$.

From (1.18) and (2.18), we get

$$
\left(\begin{array}{c}
v+\kappa \\
\kappa
\end{array}\right) B_{v, \lambda}^{(-\kappa)}=T_{2, \lambda}(v+\kappa, \kappa)
$$

where $v, \kappa$ are nonnegative positive integers.

Replacing $z$ with $\log (1+z)$ in (2.18), we get

$$
\begin{aligned}
\sum_{m=0}^{\infty} B_{m, \lambda}^{(r)} \frac{(\log (1+z))^{m}}{m !} & =\left(\frac{\log (1+z)}{(1+\lambda \log (1+z))^{\frac{1}{2 \lambda}}-(1+\lambda \log (1+z))^{-\frac{1}{2 \lambda}}}\right)^{r} \\
& =\sum_{v=0}^{\infty} D_{v, \lambda}^{*(r)} \frac{z^{v}}{v !}
\end{aligned}
$$

On the other hand,

$$
\begin{aligned}
\sum_{m=0}^{\infty} B_{m, \lambda}^{(r)} \frac{(\log (1+z))^{m}}{m !} & =\sum_{m=0}^{\infty} B_{m, \lambda}^{(r)} \sum_{v=m}^{\infty} S_{1}(v, m) \frac{z^{v}}{v !} \\
& =\sum_{v=0}^{\infty}\left(\sum_{m=0}^{v} B_{m, \lambda}^{(r)} S_{1}(v, m)\right) \frac{z^{v}}{v !}
\end{aligned}
$$

Therefore, by (2.19) and (2.20), we arrive at the following theorem.

Theorem 2.7 For $v \geq 0$, we have

$$
D_{v, \lambda}^{*(r)}=\sum_{m=0}^{v} B_{m, \lambda}^{(r)} S_{1}(v, m)
$$

Now, we introduce type 2 degenerate central poly-Bell polynomials as

$$
\operatorname{Ei}_{\kappa, \lambda}\left(\omega\left(e_{\lambda}^{\frac{1}{2}}(z)-e_{\lambda}^{-\frac{1}{2}}(z)\right)=\sum_{v=1}^{\infty} B e l_{v, \lambda}^{(\kappa, c)}(\omega) \frac{z^{v}}{v !}, \quad B e l_{v, \lambda}^{(\kappa, c)}(\omega)=0 \quad(\kappa \in \mathbb{Z}) .\right.
$$

When $\omega=1, B e l_{v, \lambda}^{(\kappa, c)}=B e l_{v, \lambda}^{(\kappa, c)}(1)$ are called type 2 degenerate poly-Bell numbers.

From (2.21), we note that

$$
\operatorname{Ei}_{\kappa, \lambda}\left(\omega\left(e_{\lambda}^{\frac{1}{2}}(z)-e_{\lambda}^{-\frac{1}{2}}(z)\right)=\sum_{m=1}^{\infty} \frac{\omega^{m}\left(e_{\lambda}^{\frac{1}{2}}(z)-e_{\lambda}^{-\frac{1}{2}}(z)\right)^{m}}{(m-1) ! n^{\kappa}}\right.
$$




$$
\begin{aligned}
& =\sum_{m=1}^{\infty} \frac{\omega^{m}\left(e_{\lambda}^{\frac{1}{2}}(z)-e_{\lambda}^{-\frac{1}{2}}(z)\right)^{m}}{(m-1) ! m^{\kappa}} \\
& =\sum_{m=1}^{\infty} \frac{\omega^{m}}{m^{\kappa-1}} \sum_{v=m}^{\infty} T_{2, \lambda}(v, m) \frac{z^{v}}{v !} \\
& =\sum_{v=1}^{\infty}\left(\sum_{m=1}^{v} \frac{\omega^{m}}{m^{\kappa-1}} T_{2, \lambda}(v, m)\right) \frac{z^{v}}{v !} .
\end{aligned}
$$

Thus, by (2.21) and (2.22), we obtain the following theorem.

Theorem 2.8 For $\kappa \in \mathbb{Z}$ and $v \in \mathbb{N}$, we have

$$
B e l_{v, \lambda}^{(\kappa, c)}(\omega)=\sum_{m=1}^{v} \frac{\omega^{m}}{m^{\kappa-1}} T_{2, \lambda}(v, m) .
$$

\section{Type $\mathbf{2}$ degenerate unipoly-Bernoulli polynomials and numbers}

The unipoly function attached to polynomials $p(\omega)$ was defined by Kim and Kim [14] as

$$
u_{\kappa}(\omega \mid p)=\sum_{v=1}^{\infty} \frac{p(v)}{v^{\kappa}} \omega^{v} \quad(\kappa \in \mathbb{Z})
$$

where $p$ denotes any arithmetic real or complex-valued function defined on $\mathbb{N}$.

Moreover,

$$
u_{\kappa}(\omega \mid 1)=\sum_{v=1}^{\infty} \frac{\omega^{v}}{v^{\kappa}}=\operatorname{Li}_{\kappa}(\omega), \quad(\text { see }[9])
$$

is the ordinary polylogarithm function.

Dolgy and Khan [4] introduced the degenerate unipoly function attached to polynomials $p(\omega)$ as follows:

$$
u_{\kappa, \lambda}(\omega \mid p)=\sum_{i=1}^{\infty} p(i) \frac{(1)_{i, \lambda} \omega^{i}}{i^{\kappa}}
$$

It is worthy to see that

$$
u_{\kappa, \lambda}\left(\omega \mid \frac{1}{\Gamma}\right)=\mathrm{Ei}_{\kappa, \lambda}(\omega), \quad(\text { see }[30])
$$

is the moderated degenerate polyexponential function.

Now, we define type 2 degenerate unipoly-Bernoulli polynomials which are given by the generating function as follows:

$$
\frac{u_{\kappa, \lambda}\left(\log _{\lambda}(1+z) \mid p\right)}{e_{\lambda}^{\frac{1}{2}}(z)-e_{\lambda}^{-\frac{1}{2}}(z)} e_{\lambda}^{\omega}(z)=\sum_{r=0}^{\infty} \beta_{r, \lambda, p}^{(\kappa)}(\omega) \frac{z^{r}}{r !}
$$

For $\omega=0, \beta_{r, \lambda, p}^{(\kappa)}=\beta_{r, \lambda, p}^{(\kappa)}(0)$ denotes type 2 degenerate unipoly-Bernoulli numbers attached to $p$. 
By (3.5), we see that

$$
\begin{aligned}
\sum_{v=0}^{\infty} \beta_{v, \lambda, \frac{1}{\Gamma}}^{(\kappa)} \frac{z^{v}}{v !} & =\frac{1}{e_{\lambda}^{\frac{1}{2}}(z)-e_{\lambda}^{-\frac{1}{2}}(z)} u_{\kappa, \lambda}\left(\log _{\lambda}(1+z) \mid \frac{1}{\Gamma}\right) \\
& =\frac{1}{e_{\lambda}^{\frac{1}{2}}(z)-e_{\lambda}^{-\frac{1}{2}}(z)} \sum_{r=1}^{\infty} \frac{(1)_{r, \lambda}\left(\log _{\lambda}(1+z)\right)^{r}}{r^{\kappa}(r-1) !} \\
& =\frac{1}{e_{\lambda}^{\frac{1}{2}}(z)-e_{\lambda}^{-\frac{1}{2}}(z)} u_{\kappa, \lambda}\left(\log _{\lambda}(1+z)\right)=\sum_{v=0}^{\infty} \beta_{v, \lambda}^{(\kappa)} \frac{z^{v}}{v !}
\end{aligned}
$$

Thus, by (3.6), we have

$$
\beta_{v, \lambda, \frac{1}{\Gamma}}^{(\kappa)}=\beta_{v, \lambda}^{(\kappa)}
$$

By making use of (3.5), we see that

$$
\begin{aligned}
\sum_{v=0}^{\infty} \beta_{v, \lambda, p}^{(\kappa)} \frac{z^{v}}{v !} & \left.=\frac{1}{e_{\lambda}^{\frac{1}{2}}(z)-e_{\lambda}^{-\frac{1}{2}}(z)} u_{\kappa, \lambda}\left(\log _{\lambda}(1+z)\right) \mid p\right) \\
& \left.=\frac{1}{e_{\lambda}^{\frac{1}{2}}(z)-e_{\lambda}^{-\frac{1}{2}}(z)} \sum_{r=1}^{\infty} \frac{p(r)(1)_{r, \lambda}}{r^{\kappa}}\left(\log _{\lambda}(1+z)\right)\right)^{r} \\
& =\frac{1}{e_{\lambda}^{\frac{1}{2}}(z)-e_{\lambda}^{-\frac{1}{2}}(z)} \sum_{r=1}^{\infty} \frac{p(r)(1)_{r, \lambda}}{r^{\kappa}}\left(\log _{\lambda}(1+z)\right)^{r} \frac{r !}{r !} \\
& =\frac{1}{e_{\lambda}^{\frac{1}{2}}(z)-e_{\lambda}^{-\frac{1}{2}}(z)} \sum_{r=1}^{\infty} \frac{p(r)(1)_{r, \lambda} r !}{r^{\kappa}} \sum_{i=r}^{\infty} S_{1, \lambda}(i, r) \frac{z^{i}}{i !} \\
& =\frac{z}{e_{\lambda}^{\frac{1}{2}}(z)-e_{\lambda}^{-\frac{1}{2}}(z)} \sum_{i=0}^{\infty} \sum_{r=1}^{i+1} \frac{p(r)(1)_{r, \lambda} r !}{r^{\kappa}(i+1)} S_{1, \lambda}(i+1, r) \frac{z^{i}}{i !} \\
& =\left(\sum_{j=0}^{\infty} B_{j, \lambda} \frac{z^{j}}{j !}\right)\left(\sum_{i=0}^{\infty} \sum_{r=1}^{i+1} \frac{p(r)(1)_{r, \lambda} r !}{r^{\kappa}(i+1)} S_{1, \lambda}(i+1, r) \frac{z^{i}}{i !}\right) \\
& =\sum_{j=0}^{\infty}\left(\sum_{i=0}^{j} \sum_{r=1}^{i+1}(j) \frac{p(r)(1)_{r, \lambda} r !}{r^{\kappa}(i+1)} S_{1, \lambda}(i+1, r) B_{j-i, \lambda}\right) \frac{z^{j}}{j !},
\end{aligned}
$$

which yields the following theorem.

Theorem 3.1 Let $j$ be a nonnegative integer and $\kappa \in \mathbb{Z}$. Then

$$
\beta_{j, \lambda, p}^{(\kappa)}=\sum_{i=0}^{j} \sum_{r=1}^{i+1}\left(\begin{array}{l}
j \\
i
\end{array}\right) \frac{p(r)(1)_{r, \lambda} r !}{r^{\kappa}(i+1)} S_{1, \lambda}(i+1, r) B_{j-i, \lambda} .
$$

Recalling from (3.5), we have

$$
\sum_{j=0}^{\infty} \beta_{j, \lambda, p}^{(\kappa)}(\omega) \frac{z^{j}}{j !}=\frac{\left.u_{\kappa, \lambda}\left(\log _{\lambda}(1+z)\right) \mid p\right)}{e_{\lambda}^{\frac{1}{2}}(z)-e_{\lambda}^{-\frac{1}{2}}(z)} e_{\lambda}^{\omega}(z)
$$




$$
\begin{aligned}
& =\frac{\left.u_{\kappa, \lambda}\left(\log _{\lambda}(1+z)\right) \mid p\right)}{e_{\lambda}^{\frac{1}{2}}(z)-e_{\lambda}^{-\frac{1}{2}}(z)} \sum_{s=0}^{\infty}(\omega)_{s, \lambda} \frac{z^{s}}{s !} \\
& =\sum_{j=0}^{\infty} \beta_{j, \lambda, p}^{(\kappa)} \frac{z^{j}}{j !} \sum_{s=0}^{\infty}(\omega)_{s, \lambda} \frac{z^{s}}{s !} \\
& =\sum_{j=0}^{\infty}\left(\sum_{s=0}^{j}\left(\begin{array}{l}
j \\
s
\end{array}\right) \beta_{j-s, \lambda, p}^{(\kappa)}(\omega)_{s, \lambda}\right) \frac{z^{j}}{j !}
\end{aligned}
$$

By Eq. (3.9), we get the following theorem.

Theorem 3.2 Let $j$ be a nonnegative integer and $\kappa \in \mathbb{Z}$. Then

$$
\beta_{j, \lambda, p}^{(\kappa)}(\omega)=\sum_{s=0}^{j}\left(\begin{array}{l}
j \\
s
\end{array}\right) \beta_{j-s, \lambda, p}^{(\kappa)}(\omega)_{s, \lambda} .
$$

From (3.5), we have

$$
\begin{aligned}
\sum_{j=0}^{\infty} \beta_{j, \lambda, p}^{(\kappa)}(\omega) \frac{z^{j}}{j !} & =\frac{1}{e_{\lambda}^{\frac{1}{2}}(z)-e_{\lambda}^{-\frac{1}{2}}(z)} u_{\kappa, \lambda}\left(\log _{\lambda}(1+z) \mid p\right)\left(e_{\lambda}(z)-1+1\right)^{\omega} \\
& =\frac{u_{\kappa, \lambda}\left(\log _{\lambda}(1+z) \mid p\right)}{e_{\lambda}^{\frac{1}{2}}(z)-e_{\lambda}^{-\frac{1}{2}}(z)} \sum_{i=0}^{\infty}(\omega)_{i} \frac{\left(e_{\lambda}(z)-1\right)^{i}}{i !} \\
& =\sum_{j=0}^{\infty} \beta_{j, \lambda, p}^{(\kappa)} \frac{z^{j}}{j !} \sum_{i=0}^{\infty}(\omega)_{i} \sum_{q=i}^{\infty} S_{2, \lambda}(q, i) \frac{z^{q}}{q !} \\
& =\sum_{j=0}^{\infty} \beta_{j, \lambda, p}^{(\kappa)} \frac{z^{j}}{j !} \sum_{q=0}^{\infty} \sum_{i=0}^{q}(\omega)_{i} S_{2, \lambda}(q, i) \frac{z^{q}}{q !} \\
& =\sum_{j=0}^{\infty}\left(\sum_{q=0}^{j} \sum_{i=0}^{q}\left(\begin{array}{l}
j \\
q
\end{array}\right)(\omega)_{i} S_{2, \lambda}(q, i) \beta_{j-q, \lambda, p}^{(\kappa)}\right) \frac{z^{j}}{j !}
\end{aligned}
$$

which yields the following theorem.

Theorem 3.3 Let $j$ be a nonnegative integer and $\kappa \in \mathbb{Z}$. Then

$$
\beta_{j, \lambda, p}^{(\kappa)}(\omega)=\sum_{q=0}^{j} \sum_{i=0}^{q}\left(\begin{array}{l}
j \\
q
\end{array}\right)(\omega)_{i} S_{2, \lambda}(q, i) \beta_{j-q, \lambda, p}^{(\kappa)} .
$$

Using (3.5), we have

$$
\begin{aligned}
\sum_{v=0}^{\infty} \beta_{v, \lambda, p}^{(\kappa)} \frac{z^{v}}{v !} & =\frac{1}{e_{\lambda}^{\frac{1}{2}}(z)-e_{\lambda}^{-\frac{1}{2}}(z)} u_{\kappa, \lambda}\left(\log _{\lambda}(1+z) \mid p\right) \\
& =\frac{1}{e_{\lambda}^{\frac{1}{2}}(z)-e_{\lambda}^{-\frac{1}{2}}(z)} \sum_{m=1}^{\infty} \frac{p(m)(1)_{m, \lambda}}{m^{\kappa}}\left(\log _{\lambda}(1+z)\right)^{m} \\
& =\frac{1}{e_{\lambda}^{\frac{1}{2}}(z)-e_{\lambda}^{-\frac{1}{2}}(z)} \sum_{m=0}^{\infty} \frac{p(m+1)(1)_{m+1, \lambda}}{(m+1)^{\kappa}}\left(\log _{\lambda}(1+z)\right)^{m+1}
\end{aligned}
$$




$$
\begin{aligned}
= & \frac{\log _{\lambda}(1+z)}{e_{\lambda}^{\frac{1}{2}}(z)-e_{\lambda}^{-\frac{1}{2}}(z)} \sum_{m=0}^{\infty} \frac{p(m+1)(1)_{m+1, \lambda} m !}{(m+1)^{\kappa}} \frac{\left(\log _{\lambda}(1+z)\right)^{m}}{m !} \\
= & \frac{\log _{\lambda}(1+z)}{e_{\lambda}^{\frac{1}{2}}(z)-e_{\lambda}^{-\frac{1}{2}}(z)} \sum_{m=0}^{\infty} \frac{p(m+1)(1)_{m+1, \lambda} m !}{(m+1)^{\kappa}} \sum_{l=m}^{\infty} S_{1, \lambda}(l, m) \frac{z^{l}}{l !} \\
= & \frac{\log _{\lambda}(1+z)}{z} \frac{z}{e_{\lambda}^{\frac{1}{2}}(z)-e_{\lambda}^{-\frac{1}{2}}(z)} \sum_{l=0}^{\infty} \sum_{m=0}^{l} \frac{p(m+1)(1)_{m+1, \lambda} m !}{(m+1)^{\kappa}} S_{1, \lambda}(l, m) \frac{z^{l}}{l !} \\
= & \sum_{v=0}^{\infty} D_{v, \lambda} \frac{z^{v}}{v !} \sum_{j=0}^{\infty} B_{j, \lambda} \frac{z^{j}}{j !} \sum_{l=0}^{\infty} \sum_{m=0}^{l} \frac{p(m+1)(1)_{m+1, \lambda} m !}{(m+1)^{\kappa}} S_{1, \lambda}(l, m) \frac{z^{l}}{l !} \\
= & \sum_{v=0}^{\infty} \sum_{j=0}^{v}\left(\begin{array}{c}
v \\
j
\end{array}\right) D_{v-j, \lambda} B_{j, \lambda} \frac{z^{v}}{v !} \sum_{l=0}^{\infty} \sum_{m=0}^{l} \frac{p(m+1)(1)_{m+1, \lambda} m !}{(m+1)^{\kappa}} S_{1, \lambda}(l, m) \frac{z^{l}}{l !} \\
= & \sum_{v=0}^{\infty}\left(\sum_{l=0}^{v} \sum_{m=0}^{l} \sum_{j=0}^{v-l}\left(\begin{array}{c}
v-l \\
j
\end{array}\right)\left(\begin{array}{c}
v \\
l
\end{array}\right)\right. \\
& \times D_{v-j-l, \lambda} B_{j, \lambda} \frac{p(m+1)(1)_{m+1, \xi} m !}{(m+1)^{\kappa}} S_{1, \lambda}(l, m) \frac{z^{v}}{v !} .
\end{aligned}
$$

Therefore, by (3.5) and (3.11), we obtain the following theorem.

Theorem 3.4 For $v \geq 0$ and $\kappa \in \mathbb{Z}$, we have

$$
\beta_{v, \lambda, p}^{(\kappa)}=\sum_{l=0}^{v} \sum_{m=0}^{l} \sum_{j=0}^{v-l}\left(\begin{array}{c}
v-l \\
j
\end{array}\right)\left(\begin{array}{l}
v \\
l
\end{array}\right) D_{v-j-l, \lambda} B_{j, \lambda} \frac{p(m+1)(1)_{m+1, \lambda} m !}{(m+1)^{\kappa}} S_{1, \lambda}(l, m) .
$$

\section{Numerical computations}

In this section, some numerical computations are done to calculate certain zeros of type 2 degenerate poly-Bernoulli polynomials and present certain interesting graphical representations. The first five members of $\beta_{v, \lambda}^{(\kappa)}(\omega)$ are obtained and given as follows:

$$
\begin{aligned}
\beta_{0, \lambda}^{(\kappa)}(\omega)= & -\frac{1}{\log 3}, \\
\beta_{1, \lambda}^{(\kappa)}(\omega)= & \frac{1}{24(\log 3)^{2}}+\frac{1}{3 \log 3}-\frac{\omega}{\log 3}, \\
\beta_{2, \lambda}^{(\kappa)}(\omega)= & -\frac{2}{729(\log 3)^{3}}-\frac{5}{72(\log 3)^{2}}+\frac{\omega}{12(\log 3)^{2}}-\frac{43}{108 \log 3}+\frac{\omega}{\log 3}-\frac{\omega^{2}}{\log 3}, \\
\beta_{3, \lambda}^{(\kappa)}(\omega)= & \frac{8}{729(\log 3)^{3}}-\frac{2 \omega}{243(\log 3)^{3}}+\frac{151}{864(\log 3)^{2}}-\frac{\omega}{4(\log 3)^{2}}+\frac{\omega^{2}}{8(\log 3)^{2}}+\frac{26}{27 \log 3} \\
& -\frac{7 \omega}{4 \log 3}+\frac{2 \omega^{2}}{\log 3}-\frac{\omega^{3}}{\log 3},
\end{aligned}
$$




$$
\begin{aligned}
\beta_{4, \lambda}^{(\kappa)}(\omega)= & -\frac{313}{6561(\log 3)^{3}}+\frac{4 \omega}{81(\log 3)^{3}}-\frac{4 \omega^{2}}{243(\log 3)^{3}}-\frac{805}{1296(\log 3)^{2}}+\frac{7 \omega}{8(\log 3)^{2}} \\
& -\frac{7 \omega^{2}}{12(\log 3)^{2}}+\frac{\omega^{3}}{6(\log 3)^{2}}-\frac{63,667}{19,440 \log 3}+\frac{31 \omega}{6 \log 3} \\
& -\frac{89 \omega^{2}}{18 \log 3}+\frac{10 \omega^{3}}{3 \log 3}-\frac{\omega^{4}}{\log 3} .
\end{aligned}
$$

Next, we present some graphs showing the behavior of $\beta_{v, \lambda}^{(\kappa)}(\omega)$ for $\kappa=4$ and $\lambda=\frac{1}{3}$; these graphs are given in Fig. 1.

The approximate solutions of $\beta_{20, \lambda}^{(\kappa)}(\omega)=0$ when $\kappa=4$ and $\lambda=\frac{1}{3}$ are calculated and displayed in Fig. 2.
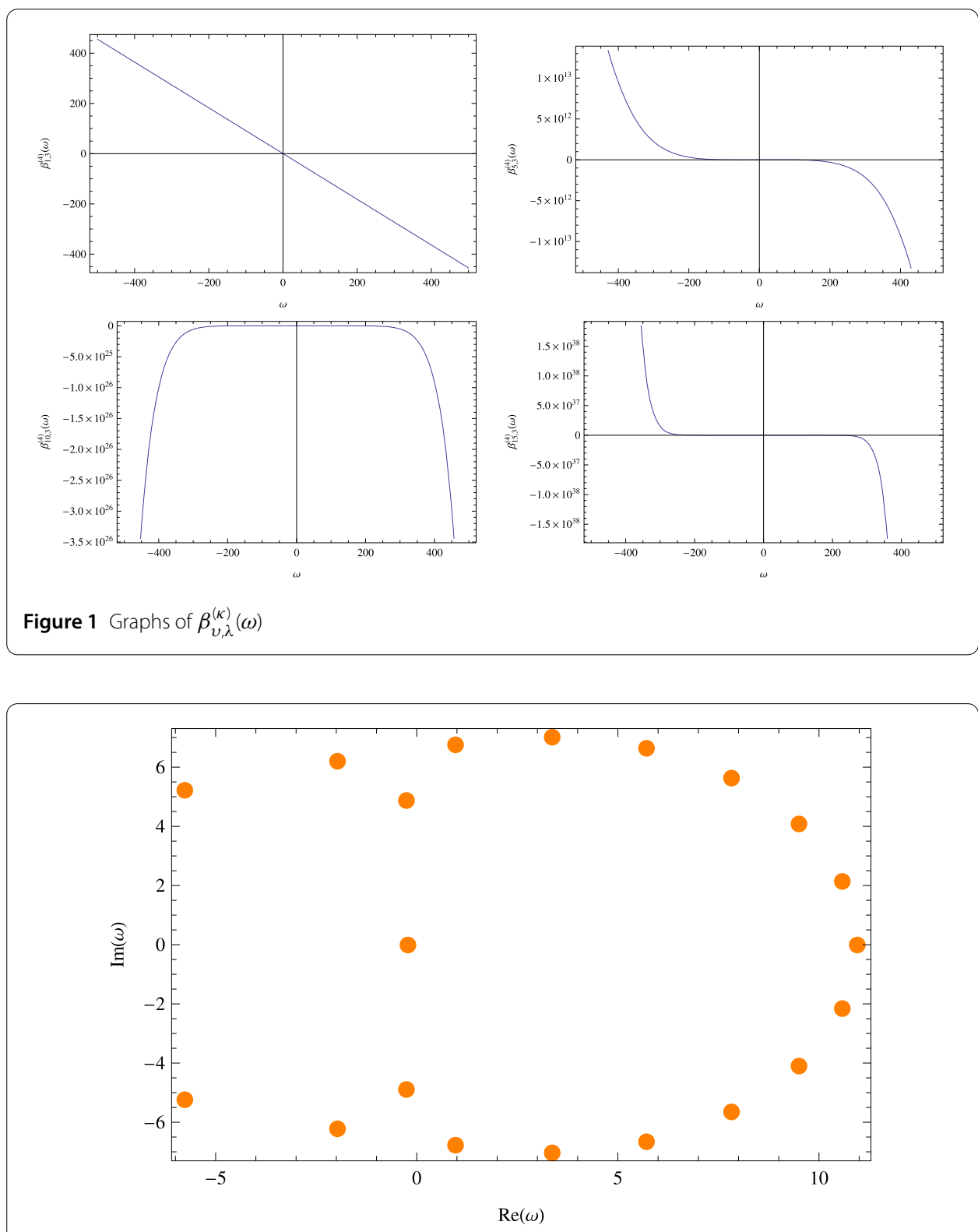

Figure 2 Zeros of $\beta_{20, \lambda}^{(\kappa)}(\omega)$ 


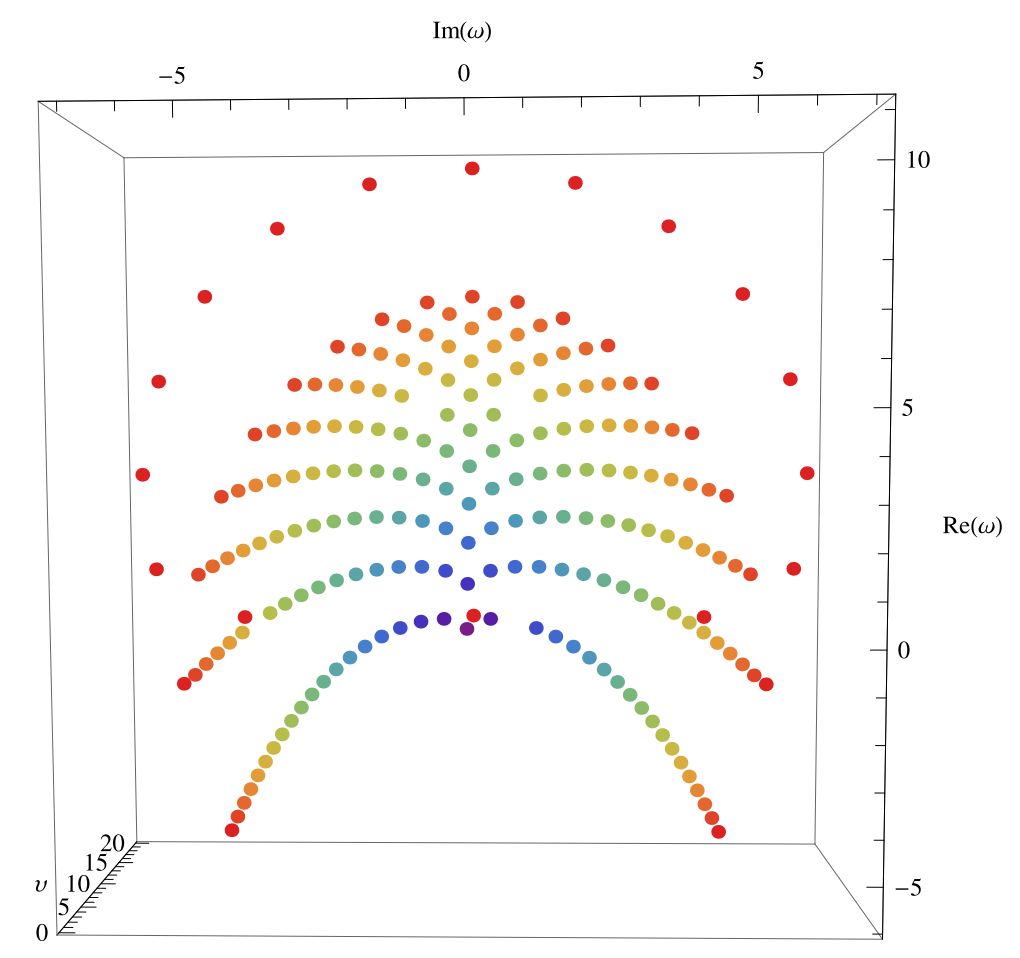

Figure 3 Stacking structure of zeros $\beta_{v, \lambda}^{(\kappa)}(\omega)$

Further, we calculate the approximate zeros of $\beta_{v, \lambda}^{(\kappa)}(\omega)=0$ for $\kappa=4, \lambda=\frac{1}{3}$ and $n=$ $1,2, \ldots, 20$, and show the stacking structure of these zeros in Fig. 3.

\section{Conclusions}

In this paper, we have studied and introduced degenerate versions of type 2 Bernoulli numbers and polynomials and derived some properties of these polynomials. We have given some relationships between higher-order Bernoulli polynomials, degenerate type 2 Bernoulli polynomials, degenerate central Bell polynomials, degenerate Stirling numbers of the first and second kind, degenerate central factorials numbers. Besides, we have introduced degenerate type 2 unipoly-Bernoulli polynomials by using degenerate unipoly polynomials and derived some identities of these polynomials. We have derived some relationship between degenerate type 2 Bernoulli polynomials and degenerate Daehee polynomials.

Acknowledgements

The authors are grateful to the anonymous referees for careful checking of the details and for helpful comments that improved this paper.

Funding

This work was supported by the Taif University Researchers Supporting Project (TURSP-2020/246), Taif University, Taif, Saudi Arabia.

Availability of data and materials

Not applicable. 
Authors' contributions

All authors contributed equally to the manuscript and typed, read, and approved the final manuscript.

\section{Author details}

'Department of Mathematics and Natural Sciences, Prince Mohammad Bin Fahd University, P.O Box 1664, Al Khobar 31952, Saudi Arabia. ${ }^{2}$ Department of Mathematics, University of Tabuk, Tabuk 71491, Saudi Arabia. ${ }^{3}$ Department of Mathematics, Hajjah University, Hajjah, Yemen. ${ }^{4}$ Department of Mathematics and Statistics, College of Science, Taif University, P.O. Box 11099, Taif 21944, Saudi Arabia.

\section{Publisher's Note}

Springer Nature remains neutral with regard to jurisdictional claims in published maps and institutional affiliations.

\section{Received: 16 June 2021 Accepted: 2 September 2021 Published online: 17 September 2021}

\section{References}

1. Carlitz, L.: A degenerate Staudt-Clausen theorem. Arch. Math. (Basel) 7, 28-33 (1956)

2. Carlitz, L.: Degenerate Stirling, Bernoulli and Eulerian numbers. Util. Math. 15, 51-88 (1979)

3. Dolgy, D.V., Jang, G.-W., Kim, T.: A note on degenerate central factorial polynomials of the second kind. Adv. Stud. Contemp. Math. (Kyungshang) 29(1), 147-159 (2019)

4. Dolgy, D.V., Khan, W.A.: A note on type two degenerate poly-Changhee polynomials of the second kind. Symmetry $13,579,1-12(2021)$

5. Hardy, G.H.: On the zeroes of certain classes of integral Taylor series. Part II.-On the integral function formula and other similar functions. Proc. Lond. Math. Soc. 2(2), 401-431 (1905)

6. Hardy, G.H.: On the zeroes certain classes of integral Taylor series. Part I.-On the integral function formula. Proc. Lond. Math. Soc. 2(2), 332-339 (1905)

7. Jang, G.-W., Kim, T.: A note on type 2 degenerate Euler and Bernoulli polynomials. Adv. Stud. Contemp. Math. (Kyungshang) 29(1), 147-159 (2019)

8. Jang, L.-C., Kim, D.S., Kim, H., Kim, T., Lee, H.: Study of degenerate poly-Bernoulli polynomials by $\lambda$-umbral calculus. Comput. Model. Eng. Sci. 2021 (2021). https://doi.org/10.32604/cmes.2021.016917

9. Kaneko, M.: Poly-Bernoulli numbers. J. Théor. Nr. Bordx. 9(1), 221-228 (1997)

10. Khan, W.A., Acikgoz, M., Duran, U.: Note on the type 2 degenerate multi-poly-Euler polynomials. Symmetry 12, 1691 (2020)

11. Khan, W.A., Ali, R., Alzobydi, K.A.H., Ahmed, N.: A new family of degenerate poly-Genocchi polynomials with its certain properties. J. Funct. Spaces 2021, Article ID 6660517 (2021)

12. Khan, W.A., Nisar, K.S., Duran, U., Acikgoz, M., Araci, S.: Multifarious implicit summation formulae of Hermite-based poly-Daehee polynomials. Appl. Math. Inf. Sci. 12(2), 305-310 (2018)

13. Khan, W.A., Sharma, S.K.: A new class of Hermite-based higher order central Fubini polynomials. Int. J. Appl. Comput. Math. 6(87), 1-14 (2020)

14. Kim, D.S., Kim, T.: A note on polyexponential and unipoly functions. Russ. J. Math. Phys. 26(1), 40-49 (2019)

15. Kim, D.S., Kim, T.: A note on a new type of degenerate Bernoulli numbers. Russ. J. Math. Phys. 27(2), $227-235$ (2020)

16. Kim, D.S., Kwon, J., Dolgy, D.V., Kim, T.: On central Fubini polynomials associated with central factorial numbers of the second kind. Proc. Jangjeon Math. Soc. 20(3), 319-331 (2017)

17. Kim, T.: A note on degenerate Stirling numbers of the second kind. Proc. Jangjeon Math. Soc. 21(4), 589-598 (2018)

18. Kim, T., Jang, L.-C., Kim, D.S., Kim, H.-Y.: Some identities on type 2 degenerate Bernoulli polynomials of the second kind. Symmetry 12(4), Article ID 510 (2020)

19. Kim, T., Kim, D.S.: A note on type 2 Changhee and Daehee polynomials. Rev. R. Acad. Cienc. Exactas Fís. Nat., Ser. A Mat. 113(3), 2783-2791 (2019)

20. Kim, T., Kim, D.S.: Degenerate central Bell numbers and polynomials. Rev. R. Acad. Cienc. Exactas Fís. Nat., Ser. A Mat. $113,2507-2513(2019)$

21. Kim, T., Kim, D.S.: Degenerate polyexponential functions and degenerate Bell polynomials. J. Math. Anal. Appl. 487(2), $124017(2020)$

22. Kim, T., Kim, D.S.: Some identities of extended degenerate $r$-central Bell polynomials arising from umbral calculus Rev. R. Acad. Cienc. Exactas Fís. Nat., Ser. A Mat. 114(1), 1-19 (2020)

23. Kim, T., Kim, D.S.: A note on central Bell numbers and polynomials. Russ. J. Math. Phys. 27(1), 76-81 (2020)

24. Kim, T., Kim, D.S.: Degenerate zero-truncated Poisson random variables. Russ. J. Math. Phys. 28(1), 66-72 (2021)

25. Kim, T., Kim, D.S., Dolgy, D.V., Lee, S.-H., Kwon, J.: Some identities of the higher-order type 2 Bernoulli numbers and polynomials of the second kind. Comput. Model. Eng. Sci. 2021 (2021). https://doi.org/10.32604/cmes.2021.016532

26. Kim, T., Kim, D.S., Jang, L.-C., Kim, H.-Y.: On type 2 degenerate Bernoulli and Euler polynomials of complex variable. Adv. Differ. Equ. 2019, 490, 15 pp. (2019)

27. Kim, T., Kim, D.S., Jang, L.-C., Lee, H., Kim, H.: Generalized degenerate Bernoulli numbers and polynomials arising from Gauss hypergeometric function. Adv. Differ. Equ. 2021, 175, 12 pp. (2021)

28. Kim, T., Kim, D.S., Kim, H.-Y., Kwon, J.: Some results on degenerate Daehee and Bernoulli numbers and polynomials. Adv. Differ. Equ. 2021, 311, 13 pp. (2020)

29. Kim, T., Kim, D.S., Kim, H.Y., Kwon, J.: Some identities of degenerate Bell polynomials. Mathematics 8(1), Article ID 40 (2020)

30. Kim, T., Kim, D.S., Kwon, J., Kim, H.-Y.: A note on degenerate Genocchi and poly-Genocchi numbers and polynomials. J. Inequal. Appl. 2020, 110, 13 pp. (2020)

31. Kim, T., Kim, D.S., Kwon, J., Lee, H.: Degenerate polyexponential functions and type 2 degenerate poly-Bernoulli numbers and polynomials. Adv. Differ. Equ. 2020, 168, 12 pp. (2020)

32. Kim, T., Kim, D.S., Kwon, J., Lee, H.: Representations of degenerate poly-Bernoulli polynomials. J. Inequal. Appl. 2021 58,12 pp. (2021) 
33. Kim, T., Kim, D.S., Kwon, J., Lee, H., Park, S.-H.: Some properties of degenerate complete and partial Bell polynomials. Adv. Differ. Equ. 2021, 304, 12 pp. (2021)

34. Kim, T., Kim, D.S., Lee, H., Jang, L.-C.: A note on degenerate derangement polynomials and numbers. AIMS Math. 6(6), 6469-6481 (2021)

35. Ma, Y., Kim, D.S., Lee, H., Kim, H., Kim, T.: Reciprocity of poly-Dedkind-type DC sums involving poly-Euler functions. Adv. Differ. Equ. 2021, 30, 18 pp. (2021)

36. $\mathrm{Ma}$, Y., Kim, T.: A note on negative $\lambda$-binomial distribution. Adv. Differ. Equ. 2021, 569, 7 pp. (2021)

37. Muhiuddin, G., Khan, W.A., Duran, U.: Two variable type 2 Fubini polynomials. Mathematics 9, 281, 1-13 (2021)

38. Muhiuddin, G., Khan, W.A., Duran, U., Al-Kadi, D.: Some identities of the multi-poly-Bernoulli polynomials of complex variable. J. Funct. Spaces 2021, Article ID 7172054 (2021). https://doi.org/10.1155/2021/7172054

39. Muhiuddin, G., Khan, W.A., Muhyi, A., Al-Kadi, D.: Some results on type 2 degenerate poly-Fubini polynomials and numbers. Comput. Model. Eng. Sci. 2021, 23 pp. (2021). https://doi.org/10.32604/cmes.2021.016546

40. Ryoo, C.S., Khan, W.A.: On two bivariate kinds of poly-Bernoulli and poly-Genocchi polynomials. Mathematics 8, 417 (2020). https://doi.org/10.3390/math8030417

41. Sharma, S.K., Khan, W.A., Araci, S., Ahmed, S.S.: New type of degenerate Daehee polynomials of the second kind. Adv. Differ. Equ. 2020, 428, 14 pp. (2020)

42. Sharma, S.K., Khan, W.A., Araci, S., Ahmed, S.S.: New construction of type 2 degenerate central Fubini polynomials with their certain properties. Adv. Differ. Equ. 2020, 587, 11 pp. (2020)

43. Sharma, S.K., Khan, W.A., Ryoo, C.S.: A parametric kind of the degenerate Fubini numbers and polynomials. Mathematics 8, 405 (2020). https://doi.org/10.3390/math8030405

\section{Submit your manuscript to a SpringerOpen ${ }^{\circ}$ journal and benefit from:}

- Convenient online submission

- Rigorous peer review

- Open access: articles freely available online

- High visibility within the field

Retaining the copyright to your article

Submit your next manuscript at $\gg$ springeropen.com 